\title{
CT in differential diagnosis of benign and malignant pleural disease
}

\author{
U. Yilmaz¹, G. Polat1 ${ }^{1}$ N. Sahin1, Ö. Soy², U. Gülay¹
}

ABSTRACT: $C T$ in differential diagnosis of benign and malignant pleural disease. U. Yilmaz, G. Polat, N. Sahin, Ö. Soy, U. Gülay.

Background. CT plays a valuable role in assessment of patients with a wide variety of diseases of the pleura, and pulmonologists should be aware of the significance of different $\mathrm{CT}$ findings for the differential diagnosis of benign and malignant pleural diseases.

Methods. 155 patients with pleural disease who had undergone CT scans of the lungs and thorax before treatment were enrolled. We retrospectively reviewed CT findings in 146 patients with proven pleural disease.

Results. Fifty-nine of the cases were malignant, 87 of them had benign pleural diseases. CT findings that were helpful in distinguishing malignant from benign pleural disease were: 1) pleural nodularity; 2) rind; 3) mediastinal pleural involvement; and 4) pleural thickening greater than $1 \mathrm{~cm}$. The sensitivities and specificities were $37 \% / 97 \%$, $22 \% / 97 \%, 31 \% / 85 \%, 35 \% / 87 \%$, respectively. CT findings differentiating malignant pleural mesothelioma from metastatic pleural disease were identified. Findings for malignant mesothelioma were as follows: 1) involvement of interlobar fissure (sensitivity $30 \%$, specificity $92 \%$ ), 2 ) pleural thickening greater than $1 \mathrm{~cm}$ (sensitivity $60 \%$, specificity $\mathbf{7 7 \%}$ ). Whereas, findings for metastatic pleural disease were mediastinal/hilar lymph node enlargement and lung parenchymal involvement $(\mathbf{P}<.05)$.

Conclusion. CT is helpful in the differential diagnosis of pleural diseases, particularly in differentiating malignant from benign conditions and metastatic pleural disease from malignant mesothelioma.

Monaldi Arch Chest Dis 2005; 63: 1, 17-22.

Keywords: Pleural disease, differential diagnosis, CT, mesothelioma.

${ }_{1}$ Department of Respiratory Medicine Chest Disease and Surgery, Training and Research Hospital, İmir,

2 Department of Radiology, Chest Disease and Surgery Training and Research Hospital, İmir, Turkey.

Correspondence: Dr. Yllmaz Ufuk, 1399 sokak No 15/11, Alsancak, 35220; İmir; Turkey; e-mail: nosigara@superonline.com drufukyilmaz@e-kolay-net

\section{Introduction}

Pleural diseases can be due to primary disorders of the mesothelial tissue, disorders of the underlying lung parenchyma, or a disease of extrathoracic origin. Malignant pleural mesothelioma (MPM), metastatic pleural disesase (MPD), empyema and tuberculous pleurisy are the most common pleural disorders in Turkey. Conventional chest radiography is used for the first evaluation of pleural diseases. Pleural effusion often remains a problem after the biochemical, bacteriologic and cyto-histological analysis of the pleural tissue and fluid. In these cases, CT plays a valuable role in the assessment of patients with a wide variety of disease of the pleura [1-3]. The most important indication for the use of $\mathrm{CT}$ is to evaluate abnormalities either seen or suspected on routine chest radiographs to establish the precise location and extent of disease especially prior to diagnostic or therapeutic procedures. But, an overlapping of the CT manifestations of the various benign and malignant pleural diseases would be expected. There are a few studies with respect to the radiological differential diagnosis of diffuse pleural disease $[4,5]$.

The aim of this study was to determine the CT features of pleural diseases by retrospective analysis which help in predicting etiology.

\section{Methods}

Between February 1995 and March 2001, 620 patients with pleural disease were admitted to the Izmir Chest Disease and Surgery Training and Research Hospital, Department. Among the 620 patients, 155 patients who had undergone CT scans of the lungs and thorax before treatment were enrolled in our study. Of these, 146 were evaluated (95 female and 51 male). Nine patients without a definite diagnosis were not evaluated. Sixty (41.1\%) patients were diagnosed with tuberculous pleurisy, $36(24.7 \%)$ as MPD, $25(17.1 \%)$ as empyema, $20(13.7 \%)$ as MPM, $3(2.1 \%)$ as lymphoma and $2(1.4 \%)$ as fibrothorax. The mean age $\pm \mathrm{SD}$ of the patients was 50.5 years \pm 1.4 .

Patients were classified into various diagnostic groups based on the following explicit criteria: 1-Malignant pleural mesothelioma; Definite diagnosis of MPM on the histological examination of pleural tissue specimens obtained by percutaneus closed biopsy of the pleura. 2-Metastatic pleural disease; Definite positive cytologic or histologic diagnosis of metastasis of the pleural space. 3-Tuberculous pleurisy; Necrotising or caseating granulomas on pleural biopsy and/or demonstration of acid-fast bacilli in pleural fluid or tissue cultures. 4-Empyema; Turbid fluid (pus) in the pleural 
space. 5-Fibrothorax; A history of an old injury or infection and dense layer of fibrous tissue over the pleural space. 6-Lymphoma; A diagnosis of lymphoma and no history of other systemic disorders, including infection, lymphocyte-predominant exudate in pleural fluid.

The patients were imaged on one of three scanners: Toshiba TCT 600 XT, Philips tomoscan AV or Hitachi scanner-pratico. Slice thickness was $10 \mathrm{~mm}$, and the patients were scanned at $10 \mathrm{~mm}$ intervals following the intravenous administration of $100 \mathrm{ml}$, nonionic contrast material. Scans were obtained from lung apices to the level of the adrenals. In some cases, it was necessary to use crosssections of 2-5 mm thickness for more a detailed examination. All of the sections were taken in supine position. All scans were photographed at window settings appropriate for lung parenchyma (window level, -800 to 900 Hounsfield units [HU]; width, 800 to $1000 \mathrm{HU}$ ) and mediastinum (window level, 0 to $25 \mathrm{HU}$; width, 350 to $400 \mathrm{HU}$ ).

The scans were assessed for the presence of pleural effusion, type of pleural thickening, involvement of interlobar fissure. The other evaluated pleural lesions were as follows: loss of volume in the involved hemithorax, unilateral or bilateral involvement, parietal pleural thickening greater than $1 \mathrm{~cm}$, pleural plaque or calcification, involvement of mediastinal and visceral pleura, contralateral mediastinal shift (CMS), and lung parenchymal involvement (such as nodule, multiple nodules, cavitary infiltration, primary tumour or consolidation, fibrosis), mediastinal/hilar lymph node enlargement (LAP), chest wall involvement.

The following criteria were used as CT definition of the pleural lesions [6,7]: 1-Regular pleural thickening was defined as a pleural thickness with smooth contour $>3 \mathrm{~mm}$. 2-Pleural nodularity was defined any degree of pleural thickening on which there were focal, irregular pleural thickenings $<4$ $\mathrm{cm}$. 3-Focal pleural mass was defined as an area of pleura-based soft tissue tumour in which the pleural thickness was $\geq 4 \mathrm{~cm}$. 4-Pleural rind was defined as a pleural thickening which envelops the entire hemithorax including the mediastinal reflection.

Involvement of the interlobar fissures (IIF) was defined as a thickening of the pleural surface of the interlobar fissure. Mediastinal pleural involvement was defined as pleural thickening bordering mediastinum. Hyaline pleural plaque was defined as a focal increase in soft-tissue density along pleura, which was well demarcated and clearly separated from the lung. Mediastinal lymph nodes were considered pathologically enlarged if they were greater than $10 \mathrm{~mm}$ in short-axis diameter in the transverse plane.

The method of study was retrospective. The CT scans were interpreted by a panel of two chest physicians and one radiologist who did not know the clinical and pathological diagnosis. A conclusion was reached by consensus.

Statistical analyses was performed using SPSS statistical software. Four groups were formed from patients in accordance with their pleural disease: (1) patients with benign pleural disease (tuberculous pleurisy plus empyema plus fibrothorax) (2) patients with malignant pleural disease (MPM plus MPD plus lymphoma) (3) patients with MPM (4) patients with MPD (included lymphoma). The comparison between patient groups (group 1 vs group 2, and group 3 vs group 4) was performed by using univariate analysis. In univariate analysis $\chi^{2}$ and Fisher's Exact test were used. A two-sided test was used at 0.05 level significance. The parameters which had $P$ values $<0.05$ in univariate analysis were also analysed according to sensitivity and specificity values.

Sensitivity and specificity values were calculated with sensitivity $(\mathrm{TP} /[\mathrm{TP}+\mathrm{FN}] \times \mathrm{x} 100)$ and specificity $(\mathrm{TN} /[\mathrm{TN}+\mathrm{FP}] \mathrm{x} 100)$. TP means the number of true positive diagnosis, TN means the number of true negative diagnosis, FP means the number of false positive diagnosis, FN means the number of false negative diagnosis.

\section{Results}

Table 1 shows the configurations of pleural thickenings and table 2 shows the distribution of CT findings among all disease groups. Table 3 shows the CT findings most suggestive of a malignant etiology (fig. 1,2). But, regular pleural thickenings were more frequently observed in benign pleural disease $(\mathrm{P}=.001)$. Regular pleural thickening was suggestive of benign cause, with a sensitivity of $48 \%$ and a spesificity of $86 \%$. Sensitivity and specificity values of CT findings that were suggestive of malignant cause were noted in table 4 .

CT findings most suggestive of MPM were pleural thickening $>1 \mathrm{~cm}$, pleural plaque, IIF $(\mathrm{P}=0.05)$. CT findings most suggestive of MPD were also mediastinal/hilar lymph node enlargement, and parenchymal involvement (table 5). All of these findings were statistically significant

Table 1. - Configurations of the pleural thickenings among all disease groups

\begin{tabular}{lcccccc}
\hline & MPM & MPD & Lymphoma & TB & Empyema & FT \\
\hline & $(\mathrm{No}=20)$ & $(\mathrm{No}=36)$ & $(\mathrm{No}=3)$ & $(\mathrm{No}=60)$ & $(\mathrm{No}=25)$ & $(\mathrm{No}=2)$ \\
\hline Rind & $7(35 \%)$ & $6(16.7 \%)$ & 0 & $2(3.3 \%)$ & $1(4 \%)$ & 0 \\
Nodularity & $10(50 \%)$ & $12(33.3 \%)$ & 0 & $3(5 \%)$ & 0 & 0 \\
Regular & $1(5 \%)$ & $4(11 \%)$ & $3(100 \%)$ & $23(38 \%)$ & $17(68 \%)$ & $2(100 \%)$ \\
Thickening size $>1 \mathrm{~cm}$ & $12(60 \%)$ & $9(25 \%)$ & 0 & $5(8.3 \%)$ & $4(16 \%)$ & $2(100 \%)$ \\
\hline
\end{tabular}

MPM; Malignant pleural mesothelioma, MPD; Metastatic pleural disease, TB; Tuberculous pleurisy, FT; Fibrothorax. 
Table 2. - Distribution of CT findings among all disease groups

\begin{tabular}{lcccccc}
\hline & MPM & MPD & Lymphoma & TB & Empyema & Fibrothorax \\
\hline & $($ No $=20)$ & $(\mathrm{No}=36)$ & $(\mathrm{No}=3)$ & $(\mathrm{No}=60)$ & $(\mathrm{No}=25)$ & $(\mathrm{No}=2)$ \\
\hline Effusion & $20(100 \%)$ & $36(100 \%)$ & $3(100 \%)$ & $60(100 \%)$ & $25(100 \%)$ & 0 \\
Plaque & $3(15 \%)$ & 0 & 0 & $3(5 \%)$ & 0 & $2(100 \%)$ \\
Calcification & $2(10 \%)$ & $2(5.6 \%)$ & 0 & $7(11.7 \%)$ & $1(4 \%)$ & $2(100 \%)$ \\
MPI & $8(40 \%)$ & $9(25 \%)$ & $1(33.3 \%)$ & $7(11.7 \%)$ & $3(12 \%)$ & 0 \\
VPI & $7(35 \%)$ & $12(33.3 \%)$ & $2(66.7 \%)$ & $9(15 \%)$ & $12(48 \%)$ & $1(50 \%)$ \\
CWI & $2(10 \%)$ & 0 & 0 & 0 & 0 & 0 \\
IIF & $6(30 \%)$ & $2(5.6 \%)$ & $1(33.3 \%)$ & $4(6.7 \%)$ & $2(8 \%)$ & 0 \\
M/H LAP & $2(10 \%)$ & $16(44.4 \%)$ & $3(100 \%)$ & $10(16.7 \%)$ & $2(8 \%)$ & 0 \\
CMS & $1(5 \%)$ & $7(19.4 \%)$ & 0 & $14(23.3 \%)$ & $1(4 \%)$ & 0 \\
PI & 0 & $18(50 \%)$ & 0 & $16(26.7 \%)$ & $11(44 \%)$ & 0 \\
Loss of volume & $8(40 \%)$ & $8(22.2 \%)$ & 0 & $6(10 \%)$ & $4(16 \%)$ & $2(100 \%)$ \\
Bilateral & $4(20 \%)$ & $14(38.9 \%)$ & $2(66.7 \%)$ & $9(15 \%)$ & $1(4 \%)$ & 0 \\
\hline
\end{tabular}

MPM; Malignant pleural mesothelioma, MPD; Metastatic pleural disease, TB; Tuberculous pleurisy, MPI; Mediastinal pleural involvement, VPI; Involvement of visceral pleura, CWI; Chest wall involvement, IIF; involvement of interlobar fissure, PI; Parenchymal involvement, CMS; Contralateral mediastinal shift, M/H LAP; Mediastinal/hilar lymph node enlargement.

Table 3. - The statistically significant CT findings in differentiation of malignant from benign pleural disease

\begin{tabular}{lccc}
\hline & $\begin{array}{c}\text { Malignant Pleural Disease } \\
(\text { No }=59)\end{array}$ & $\begin{array}{c}\text { Benign Pleural Disease } \\
(\text { No }=87)\end{array}$ & $P$ value \\
\hline Bilateral involvement & 20 & 10 & .002 \\
Pleural rind & 13 & 3 & .01 \\
Pleural nodularity & 22 & 3 & .000 \\
Regular thickening & 8 & 42 & .001 \\
Pleural thickening $>1 \mathrm{~cm}$ & 21 & 11 & .002 \\
MPI & 18 & 10 & .011 \\
M/H LAP & 21 & 12 & .003 \\
\hline
\end{tabular}

MPI; Mediastinal pleural involvement, M/H LAP; Mediastinal/hilar lymph node enlargement.

$\left(\mathrm{P}<0.05, \chi^{2}\right.$ test $)$. The parameters assessed as statistically significant findings in univariate analysis were not analysed in terms of sensitivity and specificity in differentiation of MPM than MPD except pleural thickening $>1 \mathrm{~cm}$, IIF and mediastinal/hilar lymph node enlargement. Consequently, parenchymal involvement was detected only in MPD, pleural plaque only in MPM. The presence of IIF and

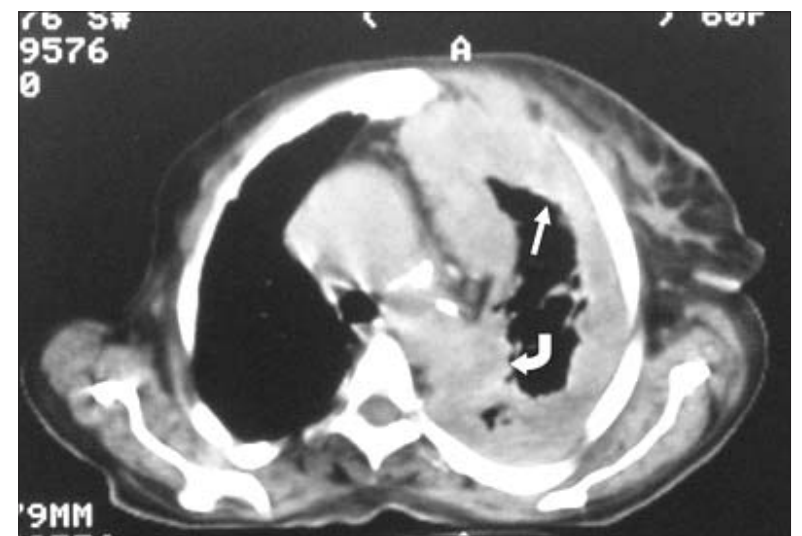

Fig. 1. - Pleural rind (arrow), pleural thickening $>1 \mathrm{~cm}$ (arrow), invasion of ascending aorta (curved arrow) in left hemithorax of a case with MPM. pleural thickening $>1 \mathrm{~cm}$ was suggestive of MPM, with a sensitivity of $30 \%$, $60 \%$ and a specificity of $92 \%, 77 \%$, respectively. The presence of mediastinal/hilar lymph node enlargement was suggestive of MPD, with sensitivity of $49 \%$ and a specificity of $90 \%$.

There were no significant differences of pleural calcification $(\mathrm{P}=0.40)$, involvement of interlobar fissure $(\mathrm{P}=0.163)$ and parenchymal involvement $(\mathrm{P}=1.00)$ between malignant and benign pleural disease. Chest wall invasion detected in only 2 cases of MPM (table 2 ). All CT features except chest wall invasion used in this study were detected in tuberculous pleurisy cases (table 2), (fig. 3).

All cases of empyema were seen as a round or lenticular fluid collection seperating slightly thick-

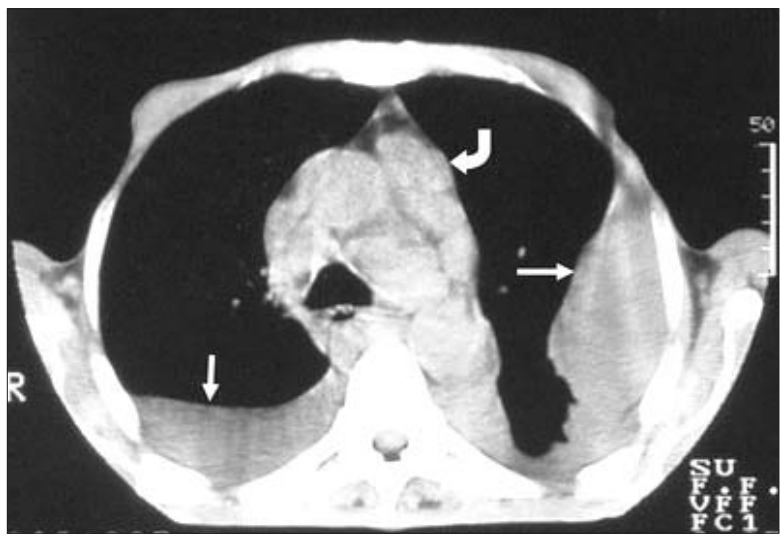

Fig. 2. - Bilateral pleural effusion (arrow), multiple mediastinal lymph node enlargement (curved arrow) in a case of lymphoma diagnosed by mediastinoscopy and cytological examination. 
Table 4. - Sensitivity and specificity of CT findings obtained by univariate analysis for the differentiation of malignant than benign pleural disease

\begin{tabular}{lcc}
\hline & Sensitivity (\%) & Specificity (\%) \\
\hline Pleural nodularity & 37 & 97 \\
Pleural rind & 22 & 97 \\
Regular thickening & 48 & 86 \\
Pleural thickening $>1 \mathrm{~cm}$ & 35 & 87 \\
MPI & 31 & 89 \\
M/H LAP & 34 & 86 \\
Bilateral involvement & 34 & 89
\end{tabular}

MPI; Mediastinal pleural involvement, M/H LAP; Mediastinal/ hilar lymph node enlargement.

Table 5. - The statistically significant CT findings in differentiation of MPM from MPD

\begin{tabular}{lccc}
\hline & MPM $(\mathrm{n}=20)$ & MPD $(\mathrm{n}=39)$ & $P$ value \\
\hline Pleural plaque & 3 & - & .035 \\
Pleural thickening $>1 \mathrm{~cm}$ & 12 & 9 & .009 \\
IIF & 6 & 3 & .05 \\
M/H LAP & 2 & 19 & .004 \\
PI & - & 18 & .000
\end{tabular}

PI; Parenchymal involvement, M/H LAP; Mediastinal/hilar lymph node enlargement, IFI; Interlober fissure involvement, MPM; Malignant pleural mesothelioma, MPD; Metastatic pleural disease.

ened visceral and parietal pleural surfaces. In 24 cases of empyema, involvement was unilateral, only in one case it was bilateral (table 1, fig. 4).

\section{Discussion}

This study suggests that pleural nodularity, rind, bilateral pleural involvement, mediastinal pleural involvement, hilar and/or mediastinal LAP can be helpful in differentiating malignant and benign pleural diseases.

Pleural response in various diseases includes three radiologically detectable manifestations: ef-

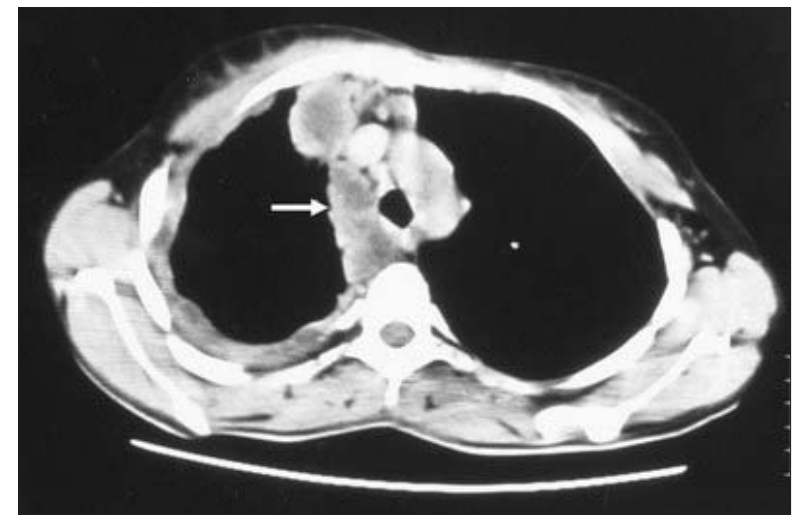

Fig. 3. - Minimal pleural effusion, thickening and rind formation (arrow), loss of volume in right hemithorax of a case with tuberculous pleurisy diagnosed by closed pleural biopsy with ultrasonography guide. Radiological improvement was achieved after treatment in this case. fusion, thickening and calcification. Pleural effusion can be the only finding with normal-appearing pleura. But, the absence of pleural thickening does not preclude a neoplastic diagnosis $[4,8]$. In our study, pleural effusion was the sole manifestation in 16 of 59 patients with malignant pleural disease.

Pleural nodularity represented a sensitivity of $37 \%$ and a specificity of $97 \%$ for malignancy in present study. Several studies have demonstrated that pleural nodularity was useful in differentiating between malignant and benign pleural diseases with $38-54 \%$ sensitivity and 94-96\% specificity but also reported that it was not enough to exclude malignancy if nodularity did not exist [4, 9-11]. Hierholzer, et al [5] also detected nodularity in 13 of 27 malignant cases, 2 of 15 benign cases. We found pleural nodularity in $3(5 \%)$ cases of tuberculous pleurisy. Aquino, et al [12] stated that $23 \%$ of cases with lymphoma and effusion had parietal pleural disease (thickening or nodules). We did not observe pleural nodularity in our cases with lymphoma.

Pleural rind was detected in 13 of 59 patients with malignant pleural disease (figure 1) as compared to only 3 of 87 benign cases (figure 3 ). In the other studies pleural rind was detected in 30-39\% of the malignant cases, and in none of the benign cases $[4,5]$. Malignant pleural disease tends to involve the entire of pleural surface, whereas reactive pleurisy usually does not affect the mediastinal pleura except tuberculous empyema (13). Due to the involvement of mediastinal pleura there is a suggested link to malignancy as demonstrated in our study $[4,5]$. However, this finding was also detected in 7 cases of tuberculous pleurisy and 3 cases of empyema in our study. Parietal pleural thickening greater than $1 \mathrm{~cm}$ was considered as a CT

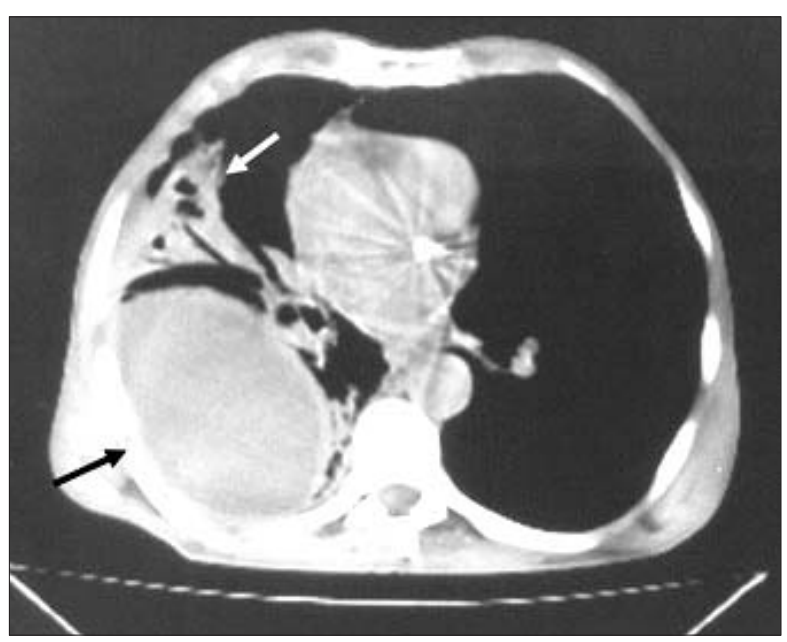

Fig. 4. - Loculated pleural effusion in lenticular form (black arrow) and middle lobe consolidation with air-bronchogram (white arrow) in case of pneumonia and empyema. 
feature for malignant pleural disease in the literature $[4,9,13]$. However, it was also reported that this feature was not important in the differantial diagnosis [5]. In our study, this feature was an important finding suggestive of malignant pleural disease.

Several studies found that the presence of pleural calcification was suggestive of benign cause with low sensitivity and high specificity [4, 5]. But in our study, calcification was present in $10 \%$ of benign cases, $6.8 \%$ of malignant cases $(\mathrm{p}>0.05)$. 'Calcified pleural plaques' were frequently seen in the patients with asbestos-related advanced benign pleural disease [9]. The different result of our study in contrast to the two studies mentioned above may be explained by the fact that we did not include the patients with benign asbestos pleural effusion. Bilateral pleural involvement was a more common finding in malignant pleural diseases than benign, especially in cases of lymphoma and metastatic pleural disease. Leung, et al [4] reported that bilateral pleural involvement was not useful in differentiating malignant from benign pleural diseases in contrast to our study. This could be explained by the existence of tuberculous pleurisy cases which was unilateral in a high number in contrast to the study of Leung, et al which did not include tuberculous pleurisy.

MPM is a rare neoplasm but, pleural metastases are the most common pleural neoplasm. In spite of all diagnostic studies, the differantial diagnosis can remain problematic. Leung, et al [4] reported that, CT was not helpful in differentiation of MPM and MPD. In the present study, the CT findings suggestive of MPM were pleural plaque, involvement of interlobar fissure $(\mathrm{P}=.05)$, pleural thickening greater than $1 \mathrm{~cm}$, whereas findings suggestive of MPD were lung parenchymal changes, contralateral mediastinal shift and mediastinal and/or hilar LAP.

Pleural plaque was detected only in cases of MPM among all malignant cases in our study. Pleural plaque was found in $15-27 \%$ of cases with MPM and 3-12.5\% of cases with metastatic disease as cited in the literature $[4,9]$. Involvement of interlobar fissure appears as a thickening and/or nodularity. These findings look like thickening seen on other pleural surfaces [14]. Involvement of interlobar fissure was seen in $30 \%$ of cases with MPM, $5.6 \%$ of MPD. In the our other study, interlobar fissure involvement was detected in 54\% of cases with MPM [6]. In the study of Leung, et al [4], interlobar fissure involvement was observed in $63.6 \%$ of cases with MPM, $45.8 \%$ of cases with MPD. Pleural thickening greater than $1 \mathrm{~cm}$ was seen in $70-94 \%$ of the cases MPM [6, 15]. Metintas et al reported that this feature had importance for differentiation of MPM from MPD with a sensitivity $59 \%$ and a specificity $82 \%$ in multivariate analysis [9].

In our study, lung parenchymal involvement was present in $50 \%$ of cases with MPD. We found only compressive changes in patients with MPM. Because it was a relatively non-specific finding, we did not evaluate as parenchymal involvement. However, in the study of Leung, et al [4], involvement of the lung parenchyma was present in 8 of the 11 cases of MPM and 21 of the 24 cases of MPD.

Mediastinal and/or hilar lymphadenopathy was identified in all cases of lymphoma, 44.4\% of metastatic pleural disease and only 10\% of MPM and it was helpful in differentiating MPD from MPM. Correlating the CT findings with the surgical findings has shown that it caused an underestimation of mediastinal nodal involvement by tumour [16]. As a result this conclusion should have limited value. Adams, et al [17] suggested that the presence of hilar LAP may be helpful in differentiating metastases from MPM. Leung, et al [4] identified hilar LAP in only 2 of the 39 malignant cases and suggested that, CT findings of MPM and MPD are similar and could not be used in the differentiation confidently.

Invasion of chest wall is suggestive of malignancy but have a low sensitivity. In our study, it was present in only 2 cases of MPM. It was not helpful in the differentiation of pleural diseases. In the studies of Leung, et al [4] and Rusch, et al [18], it was stated that chest wall invasion was not helpful in the differentiation of MPM from other pleural malignities.

Typical CT finding for empyema are being shape of lenticular form and impression on bronchial and pulmonary arteries by pushing parenchyma [19]. In our study, pleural thickening was not helpful for differentiation of empyema from other pleural diseases. Extrapleural subcostal tissue was not evaluated in the present study. This is a limitation of our study. Aquino, et al [10] detected pleural thickenings in all cases of empyema. Waite, et al [20] also reported that CT can not be helpful to differentiate empyema from other pleural diseases.

Magnetic resonance imaging, although not commonly performed, can occasionally be useful in differentiating benign from malignant pleural disease [21]. The use of Positron Emission Tomography (PET) is still being evaluated in patients with suspected malignant pleural effusions; it appears to be more useful in patients with a focal or genarilised pleural thickening. PET discriminates poorly between infective and malignant causes, and low-grade malignancy, as in patients with fibrous mesothelioma and small volume disease, is difficult to identify [22, 23].

The results of this study are subject to bias. The retrospective selection of patients results inherently in selection bias. Despite the inherent shortcomings of a retrospective study design, the present study demonstrated that 'pleural nodularity', 'pleural rind' each are CT features for differentiation of malignant from benign pleural disease with very high specifities. The CT findings suggestive of MPM are pleural plaques, involvement of interlobar fissure, pleural thickening $>1 \mathrm{~cm}$ whereas lung parenchymal involvement, and hilar and/or mediastinal LAP suggest metastatic pleural disease. 


\section{References}

1. Kreel L. Computed tomography of the lung and pleura. Semin Roentgenol 1978; 13: 213-225.

2. Kreel L. Computed tomography in mesothelioma. Semin Oncol 1981; 8: 302-312.

3. McLoad TC. CT and MR in pleural disease. Clin Chest Med 1998; 19: 261-276.

4. Leung AN, Muller NL, Miller RR. CT in differential diagnosis of diffuse pleural disease. AJR 1990; 154: 487492.

5. Hierholzer J, Luo L, Bittner RC, et al. MRI and CT in the differential diagnosis of pleural disease. Chest 2000; 118: 604-609.

6. Yılmaz U, Utkaner G, Yalnız E, Kumcuoğlu Z. Computed tomographic findings of environmental asbestosrelated malignant pleural mesothelioma. Respirology 1998; 3: 33-38.

7. Yilmaz U, Kumcuoğlu Z, Utkaner G, et al. Computed tomography findings of tuberculosis pleurisy. Int $J$ Tuberc Lung Dis 1998; 2: 164-167.

8. Maffessanti M, Tommase M, Pellegrini N. Computed tomography of free pleural effusions. Eur J Radiol 1987; 7: 87-90.

9. Metintas M, Ucgun I, Elbek O, Erginel S, et al. Computed tomography features in malignant pleural mesothelioma and other commonly seen pleural diseases. Eur J Radiology 2002; 41 (1): 1-9.

10. Aquino SL, Webb WR, Gushiken BJ. Pleural exudates and transudates: Diagnosis with contrast-enhaced CT. Radiology 1994; 192: 803-808.

11. Arenas-Jimenez J, Alonso-Charterina S, Sanchez-Paya $\mathrm{J}$, et al. Evaluation of CT findings for diagnosis of pleural effusions. Eur Radiol 2000; 10 (4): 681-690.

12. Aquina SL, Chen MY, Kuo WT, et al. The CT ap- pearence of pleural and extrapleural disease in lymphoma. Clin Radiol 1999; 54 (10): 647-650.

13. Müller NL. Imaging the pleura. Radiology 1993; 186: 297-309.

14. Kawashima A, Libshitz HI. Malignant Pleural Mesothelioma. CT manifestations in 50 case. AJR 1990; 155: 965-969.

15. Ng CS, Munden RF, Libshitz HI. Malign pleural mesothelioma: the spectrum of manifestations on CT in 70 cases. Clin Radiol 1999; 54: 415-421.

16. Rusch VW, Godwin JD, Shuman WP. The role of computed tomography scaning in the initial assessment and the follow-up of malignant pleural mesothelioma. $J$ Thorac Cardiovasc Surg 1988; 96: 171-177.

17. Adams VI, Unni KK, Muhm JR, et al. Diffuse malignant mesothelioma of pleura. Cancer 1986; 58: 15401551.

18. Rusch VW, Godwin JD, Shuman WP. The role of computed tomography scanning in the initial assessment and the follow-up of malignant pleural mesothelioma. $J$ Thorac Cardiovasc Surg 1988; 96: 171-177.

19. Stark DD, Federle MP, Goodman PJ, et al. Differentiating lung abscess and empyema radiography and computed tomography. AJR 1983; 141 (1): 163-167.

20. Waite RJ, Carbonneau RJ, Balikian JP. Parietal pleural changes in empyema:Appearences on CT. Radiology 1990; 175: 145-150.

21. Evans AL, Gleeson FV. Radiology in pleural disease: State of the art. Respirology 2004; 9: 300-312.

22. Burry T, Paulus P, Dowlatt A, et al. Evaluation of pleural disease with FDG PET imaging; preliminary report. Thorax 1997; 52: 187-189.

23. Duysinx B, Nguyen D, Louis R, et al. Evaluation of pleural disease with 18 -fluorodeoxyglicose positron emission tomography imaging. Chest 2004; 125: 489-493.

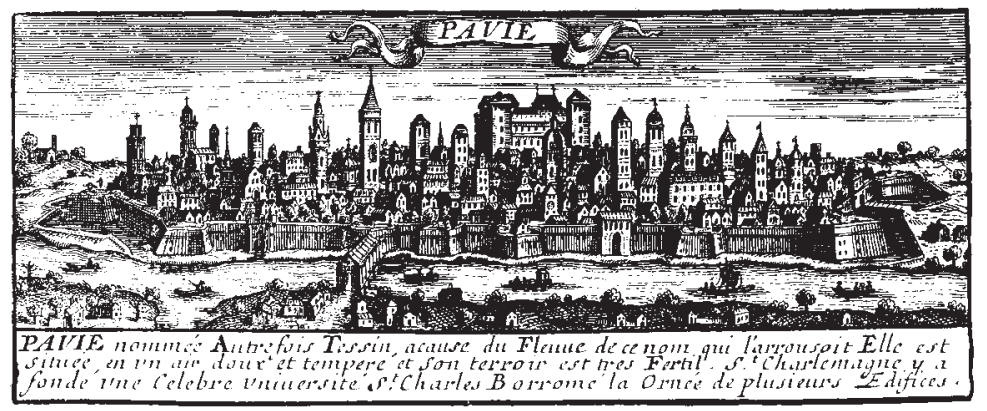

